\title{
Regulation of blood viscosity in disease prevention and treatment
}

\author{
CHEN Gan ${ }^{1}$, ZHAO Lian ${ }^{1}$, LIU YaoWen ${ }^{3}$, LIAO FuLong ${ }^{2}$, HAN Dong $^{2} \&$ ZHOU Hong ${ }^{1 *}$ \\ ${ }^{1}$ Institute of Transfusion Medicine, Academy of Military Medical Sciences, Beijing 100850, China; \\ ${ }^{2}$ National Center for Nanoscience and Technology, Beijing 100080, China; \\ ${ }^{3}$ Institute of Pharmacology and Toxicology, Academy of Military Medical Sciences, Beijing 100850, China
}

Received May 31, 2011; accepted October 19, 2011

\begin{abstract}
Blood viscosity plays an important role in maintaining vascular homeostasis. Under normal physiological conditions, blood viscosity is kept at a relatively stable level by a variety of regulatory mechanisms. However, under pathological conditions, disorders in these regulatory mechanisms mediated by endothelial cell dysfunction result in the pathogenesis and development of certain diseases, such as cardio-cerebrovascular diseases, diabetes mellitus, and hemorrhagic shock. Therefore, monitoring and regulating blood viscosity are important for the diagnosis, treatment, and prognosis of disease. This article reviews the role of blood viscosity regulation and its importance in disease prevention and treatment.
\end{abstract}

blood viscosity, cardio-cerebrovascular diseases, diabetes mellitus, hemorrhagic shock, endothelial dysfunction

Citation: Chen G, Zhao L, Liu Y W, et al. Regulation of blood viscosity in disease prevention and treatment. Chin Sci Bull, 2012, 57: 1946-1952, doi: $10.1007 / \mathrm{s} 11434-012-5165-4$

Viscosity, a basal parameter in rheology, is a major determinant of the shear stress imparted by blood flow, and plays an important role in maintaining vascular homeostasis. Blood viscosity is mainly determined by hematocrit levels, plasma viscosity, and the deformability and aggregation of red blood cells (RBCs). Blood viscosity varies under normal physiological conditions due to differences in heredity, sex, and geography. Under pathological conditions, changes in blood viscosity usually correlate with endothelial cell dysfunction and changes in the shear stress imparted by blood flow, which can damage the circulatory system and related tissues and organs. Abnormal blood viscosity plays an important role in the development of cardio-cerebrovascular disease, diabetes, hemorrhagic shock, and renal disease. When treating these diseases, much attention is paid to the regulation of blood viscosity, which is pivotal to successful treatment and avoiding undesirable syndromes. Since the 1970 s, researchers have paid greater attention to the role of hemorheological parameters, including blood viscosity, in the diagnosis and treatment of diseases [1]. In the 1980s, the

*Corresponding author (email: zhouhtt@yahoo.com.cn) relationship between hemorheology and disease was intensively investigated and several monographs were published $[2,3]$. Therefore, this article will review recent progress regarding the manipulation of blood viscosity for disease prevention and treatment.

\section{Blood viscosity under physiological conditions}

Within the physiological range of shear rates, blood viscosity is primarily determined by hematocrit levels and plasma viscosity [4]. The deformability and aggregation of erythrocytes and the interaction between blood cells are also important contributing factors. The viscosity of plasma, which has similar properties to those of a Newtonian fluid, is determined mainly by the concentration of plasma proteins, but the erythrocyte deformability and aggregation vary with different blood shear rates. Therefore, blood has the properties of a pseudoplastic non-Newtonian fluid. In addition to plasma and hematocrit, erythrocyte deformability at high shear rates $\left(>200 \mathrm{~s}^{-1}\right)$ is a major determinant of blood viscosity. At low shear rates, blood viscosity is affected by 
deformability and aggregation of erythrocytes and by platelet aggregation [5,6].

Epidemiological studies show that blood viscosity is affected by many factors. A study conducted in apparently normal adults by De Simone et al. [7] found that cholesterol and triglyceride levels were positively related to blood viscosity; obesity, high sodium intake and aging also increased blood viscosity. Mental stress may also induce the decrease in erythrocyte deformability and an acute elevation in hematocrit levels $[6,8,9]$. Blood viscosity is also influenced by gender. Men have a significantly higher blood viscosity than women due to differences in hematocrit and the deformability and aggregation of erythrocytes $[7,8,10]$. Geographical factors also affect blood viscosity. Compared with low-altitude residents, high altitude-residents have higher levels of hematocrit, blood viscosity [11-13], blood flow, and circulating nitric oxide (NO) products [14]. A study by Cheng et al. [15] indicates that, although hematocrit is higher among high-altitude residents, compensatory regulation can prevent excessive increases in blood viscosity via increased erythrocyte deformability and decreased erythrocyte aggregation. Martini et al. [16] suggest that high altitudes cause increased hematocrit levels and, therefore, increased blood viscosity, which results in increased shear stress on the endothelium; this, in turn, causes the release of stress-mediated vasodilators, leading to a reduction in blood pressure. This may be one of the explanations for the low mortality from coronary heart disease and stroke observed in high altitude residents [17].

Under normal physiological conditions, variations in blood viscosity directly affect the endothelium, which then regulates blood viscosity and blood vessel diameter by releasing vasoactive factors to keep the vascular wall shear stress relatively stable. Because of these endothelium-dependent regulatory mechanisms, increased blood viscosity does not result in increases vascular resistance. In a large-scale epidemiological study, De Simone et al. [18] found that blood viscosity was negatively related to pulse pressure in apparently normal adults. A study by Salazar Vázquez et al. $[19,20]$ showed that mean arterial blood pressure (MAP), which reflects peripheral vascular resistance, was negatively correlated with blood viscosity in healthy children and premenopausal women, but this was not significant. However, some research suggests that, hematocrit levels and blood viscosity in normal adults do not correlate with MAP [21].

\section{Abnormalities in blood viscosity and disease}

Abnormal changes in blood viscosity play an important role in the pathogenesis and development of many diseases, including cardio-cerebrovascular diseases, diabetes mellitus, and hemorrhagic shock. Abnormal changes in blood viscosity and endothelial dysfunction promote each other, forming a vicious circle. Endothelial cell dysfunction, a key link in the pathogenesis of many diseases, is associated with imbalances in the production and release of vasoactive factors, such as vasoconstrictors and vasodilators, pro-coagulant and anti-coagulant factors, and inhibitors and promoters of proliferation [22].

\subsection{Abnormalities in blood viscosity and cardio- cerebrovascular diseases}

Increased blood viscosity is associated with cardio-cerebrovascular diseases. In a 5-year follow-up study, Lowe et al. [23] found that blood viscosity and plasma viscosity were significantly higher in patients who experienced cardiovascular events than in those who did not, and that was because of increases in fibrinogen concentration and hematocrit. In a similar study, Lee et al. [24] showed that increased blood viscosity was associated with the early stages of atherosclerosis. In addition, hyperviscosity may play a role in the occurrence of cerebral ischemic complications in patients with atrial fibrillation (AF). AF patients who had experienced previous cerebral ischemic events had a higher blood viscosity than AF patients who had not experienced such events [25].

Abnormal increases in blood viscosity are associated with the development and prognosis of cardio-cerebrovascular diseases. Increasing hematocrit, abnormal increases in fibrinogen concentration, and blood viscosity can all make cardio-cerebrovascular diseases worse and increase the long term risk of mortality [26,27]. Cecchi et al. [28] found that blood viscosity was independently and positively associated with infarct size. It is possible that increased blood viscosity may contribute to a lack of myocardial perfusion, which may then result in an increased infarct size. Malinova et al. [29] undertook a 7-year follow-up study in patients with ischemic heart disease and suggested that blood viscosity and blood flow change with the severity of coronary artery disease. In hypertensive men, increased blood viscosity at low shear rates is a predictor of cardiovascular events, including acute myocardial infarction, unstable angina, or stroke [30].

In cardio-cerebrovascular diseases, abnormal increases in blood viscosity caused by factors such as an increase in oxygen/nitrogen free radicals, fibrinogen, and oxidized low density lipoprotein will not only increase platelet adhesion, but also alter vascular wall shear stress. This leads to endothelial dysfunction, increased inflammation, and hypertrophy of smooth muscle cells [31], as well as vascular stenosis and insufficient blood flow, which can lead to myocardial infarction and stroke.

\subsection{Abnormalities in blood viscosity associated with diabetes mellitus}

Increased blood viscosity is closely related to the occurrence and development of diabetes mellitus. Insulin resistance and hyperinsulinemia predispose to diabetes mellitus. Increased 
blood viscosity will, in turn, impair blood flow within the tissues and limit the delivery of insulin, glucose, and oxygen to metabolically active tissue [32], ultimately resulting in increased insulin resistance [33]. Hyperinsulinemic obese female patients show significantly higher plasma viscosities than normoinsulinemic patients [34]. Increased blood viscosity in patients with diabetes mellitus is an important factor that contributes to diabetic macrovascular complications $[19,35,36]$. Blood viscosity in diabetic patients is also positively correlated with the severity of retinopathy $[37,38]$. Diabetic patients with retinopathy show higher blood viscosity than diabetics without retinopathy at both high and low shear rates [37]. However, another study came to the opposite conclusion [39]. Blood viscosity in diabetic patients who have experienced complications, such as microalbuminuria and nephropathy, is also higher than that in patients without such complications [40,41]. Measurements of blood viscosity at low shear rates in diabetic patients have prognostic value. Compared with those who were either asymptomatic or had mild peripheral vascular disease, patients with critical limb ischemia showed higher blood viscosity and RBC aggregation at low shear rates [42].

Diabetes mellitus is accompanied by decreased production of the vasodilators, NO, and prostacyclin, and increased formation of vasoconstrictor eicosanoids [43], leading to increased peripheral vascular resistance and abnormal blood pressure. This is due to impairment of endothelial vasodilator mechanisms. Salazar Vázquez et al. [21] found a correlation between MAP and hematocrit in patients with a previous diagnosis of type 2 diabetes. MAP levels decrease significantly as hematocrit levels increase within the low hematocrit range $(<43 \%)$, but increase as hematocrit levels increase within the high hematocrit range ( $>43 \%)$. Nevertheless, blood viscosity in children with type 1 diabetes is positively correlated with MAP, indicating endothelial dysfunction [19].

Many factors contribute to increased blood viscosity in diabetic patients. First, increased glucose causes endothelial dysfunction and, therefore, disorders in the feedback mechanisms that regulate blood viscosity. Second, high glucose levels increase the generation of active oxygen species in erythrocytes and endothelial cells, leading to reduced RBC deformability and increased blood viscosity. Third, increased RBC aggregation [44] and fibrinogen levels also contribute to increased blood viscosity in diabetic patients. Microcirculatory disturbances caused by abnormal changes in blood viscosity may worsen the complications associated with diabetes mellitus $[45,46]$.

\subsection{Abnormalities in blood viscosity associated with other diseases}

Increased blood viscosity is also observed in other diseases. Plasma viscosity, fibrinogen concentration, and RBC aggregation in renal transplant recipients are all significantly higher than those in control subjects [47]. Elevated blood viscosity in patients with systemic lupus erythematosus may be associated with atherothrombotic disease [48]. In addition, increased blood viscosity is an important factor that impacts upon prognosis [49]. As a marker of vasculopathy, increased blood viscosity may accelerate disease progression in Alzheimer's disease patients by worsening vasculopathy [50].

In some diseases, such as chronic anemia and liver disease, plasma and blood viscosity show a long term decline because of low concentration of plasma proteins rather than an abnormal increase in blood viscosity [51-53], leading to disorders in endothelial shear response mechanisms. This may also occur in hemoglobin or RBC diseases [54]. During the early stage of severe hemorrhagic shock, a sharp decline plasma and blood viscosity occurs resulting from the loss of RBCs and plasma proteins [55]. With respect to the microcirculation, a rapid decrease in arteriolar blood flow and functional capillary density during hemorrhagic shock may give rise to microcirculatory disturbances $[56,57]$.

\section{Regulation of blood viscosity and treatment of disease}

As previously mentioned, abnormal changes in blood viscosity are closely related to the pathogenesis and development of many diseases. There is growing evidence to suggest that regulation of blood viscosity is beneficial for disease treatment. Hemodilution can be used to treat diseases resulting from abnormal increases in blood viscosity. However, under a severe decrease in blood viscosity, e.g. hemorrhagic shock and extreme hemodilution, the appropriate increase in blood viscosity can maintain the shear stress on endothelium and maintain vasodilatation, thereby improve microcirculation and increase cardiac output [58].

\subsection{Treatments that reduce blood viscosity}

For diseases associated with abnormal increases in blood viscosity, such as cardio-cerebrovascular diseases and diabetes mellitus, two methods are currently used to reduce viscosity: direct and indirect. Direct methods include plasma exchange, phlebotomy, and rheopheresis. Plasma exchange uses plasma substitutes to replace plasma to reduce blood viscosity without affecting the packed cell volume. In the short term, it reduces blood viscosity, the concentration of fibrinogen, and the levels of triglycerides and cholesterol, and improves local blood flow [59,60]. Phlebotomy, which is used to treat patients with polycythemia vera and hemochromatosis, reduces hematocrit levels, and plasma and blood viscosity via controlled bleeding [61,62]. The combination of phlebotomy and fluid therapy has been used to treat diseases such as ischemic stroke and myocardial infarction. Strand et al. [63] used phlebotomy and repeated 
low-molecular weight dextran infusions to treat the early phase of ischemic stroke. During the 3-month follow-up, the treatment significantly reduced blood viscosity and improved the overall clinical outcome of patients. Rheopheresis therapy improves hemorheological parameters by reducing fibrinogen levels, low-density lipoprotein cholesterol, and alpha-2-macroglobulin and improves the treatment of microvascular diseases, and relieves the symptoms of agerelated macular degeneration and peripheral arterial occlusive disease [64].

Indirect therapy reduces blood viscosity by regulating the factors that influence blood viscosity, such as erythrocytes, platelets, and endothelial cells. For example, antioxidant therapy uses vitamins $\mathrm{E}$ and $\mathrm{C}$ to reduce oxidative stress in vivo, increase RBC deformability, improve endothelial dysfunction, and lower blood viscosity [65-67]. In a 6-month follow-up study, Galduroz et al. [10] found that the extract of Ginkgo biloba significantly reduced blood viscosity. Nitroglycerin can also reduce blood viscosity, the decrease being associated with an increase in myocardial blood flow and a decrease in vascular resistance [68]. In addition, the essence of stimulating the circulation to end stasis in Chinese Medicine is similar to reducing blood viscosity to improve microcirculation. Traditional Chinese medicines, such as Salvia miltiorrhiza Bunge and Ligusticum chuanxiong Hort, effectively regulate blood viscosity by protecting the endothelium and inhibiting platelet aggregation [69-73].

\subsection{Treatments that increase blood viscosity}

Blood viscosity shall be increased moderately when treating diseases that extremely reduce viscosity. Transfusion is commonly used to restore blood volume and improve the delivery of oxygen and nutrients. Recent studies interpreted the effects of transfusion in terms of maintaining blood viscosity. The studies concluded that transfusion works by maintaining viscosity and restoring oxygen-carrying capacity. Transfusion restores blood viscosity, and maintains the shear stress on the endothelium and microvascular perfusion such that oxygen delivery and removal of metabolites can be maintained [58,74-76]. Martini et al. [77] studied exchange transfusions of homologous packed red blood cells using the awake hamster window chamber model, and observed a moderate elevation of hematocrit (10\% above baseline), which causing an increased cardiac index, increased oxygen transport and consumption, and a reduction in total peripheral vascular resistance.

Maintenance of blood viscosity should also be taken into consideration when developing blood substitutes. Researchers found that some hemoglobin-based oxygen carriers caused transient systemic hypertension [78,79] and reduced cardiac output [80]. This is because large infusions of blood substitutes lower blood viscosity and the shear stress on the endothelium, which down-regulates the production of vasodilators by endothelial cells, causing vasoconstriction and increasing total peripheral vascular resistance [81]. In the hemorrhagic shock model, viscous blood substitutes limit vasoconstriction $[82,83]$ and improve functional capillary density and oxygen content.

Maintenance of plasma viscosity is critically important when rapid infusion of erythrocytes and increased blood viscosity cannot be performed. Tsai et al. [84,85] showed that the use of high viscosity plasma expanders during extreme hemodilution could maintain functional capillary density and significantly improve capillary perfusion. The researchers concluded that increased production of vasodilators by the endothelium (e.g. NO) might be the major reason for improvements in the microcirculation. Using the severe experimental hemorrhagic shock model based on the infusion of hypertonic saline, Golden Syrian Hamsters were randomly infused with Hextend $(6 \%$ Hetastarch in lactated electrolyte solution, $4 \mathrm{mPa}$ s) or viscosity-enhanced Hextend (Hextend $+0.4 \%$ alginate, $8 \mathrm{mPa} \mathrm{s}$ ). Resuscitation with Hextend resulted in higher MAP, but viscosity-enhanced Hextend showed better results in terms of improved functional capillary density, microvascular perfusion, and the restoration of acid-base balance [86].

\section{Regulation of blood viscosity}

Although blood viscosity plays an important role in disease prevention and treatment, research into the mechanisms that regulate blood viscosity is still lacking. Previous studies show that the endothelium plays a key role in regulating blood viscosity. Endothelium, as both the sensor and effector of blood viscosity, not only is regulated by blood viscosity [87], but also plays a vital role in the feedback regulation mechanisms that control it. The effects of blood viscosity on an organism depend on endothelial cell function. Under normal physiological conditions, increased blood viscosity leads to increased vascular wall shear stress and increased production of endothelial-derived relaxing factors, such as NO and prostacyclin. These vasoactive substances not only cause vasodilatation and, therefore, lower MAP, but also reduce blood viscosity by improving RBC deformability, thereby suppressing RBC aggregation and platelet aggregation and adhesion [88-90]. Under pathological conditions, many harmful factors, such as mental stress, inflammation [27] and high glucose levels, result in increased production of reactive oxygen species, fibrinogen, immunoglobulins, and hematocrit, which lead to abnormally increased blood viscosity. Furthermore, they may both directly and indirectly cause endothelial dysfunction and feedback regulation mechanism disorders. Therefore, abnormal changes in blood viscosity and endothelial dysfunction promote each other and form a vicious circle.

Blood viscosity is closely related to endothelial cell function. Malek et al. [91] found that a constant, high level of shear stress which generated by the flow and viscosity could 
suppress the expression of endothelin-1 in bovine aortic endothelial cells. Koller et al. [92] varied the viscosity of the perfusate in isolated arterioles from the cremaster muscle by adding dextran, and found that, at a constant flow rate, increases in the viscosity of the perfusate caused gradual vasodilation. Viscosity-induced dilation was eliminated by removing the endothelium from the arterioles, confirming that viscosity-induced dilation is endothelium-dependent. They concluded that increased perfusate viscosity increased shear stress and, thus, induced prostacyclin release by the endothelium, resulting in vasodilation. Using the severe hemodilution model, Bertuglia [93] found that hyperviscous infusions preserved both the endothelium and capillary perfusion.

As mentioned previously, viscosity can affect endothelial cell function by altering shear stress. Furthermore, substrate stiffness and micro- and nano-structural surfaces can directly modulate cell shape, stiffness, gene expression, and adhesion molecule distribution [94-96]. It is interesting to speculate whether blood viscosity, as one determinant of the viscoelasticity of the material that contacts the endothelium, may modulate endothelial cell shape, stiffness, gene expression, adhesion molecule distribution and, therefore, overall health status, in addition to directly affecting endothelial cells through shear stress. This would constitute an important additional endothelium-dependent regulatory mechanism of blood viscosity and needs further study.

\section{Conclusions and expectations}

Blood viscosity is an important parameter to be considered during drug evaluation and research into their mechanisms of action as it correlates closely with the pathogenesis, development, and prognosis of cardio-cerebrovascular diseases, diabetes and other diseases. More interest is now being paid to treatments that manipulate blood viscosity. However, the bio-effectiveness of viscosity is sometimes overlooked in the clinic. The role of hemorheology in the pathogenesis, development, and prognosis of disease needs more attention. Future investigations should include the following: (1) The relationship between hemorheological parameters and the correlation between abnormal blood viscosity and endothelial cell dysfunction, such as blood viscosity, and the pathogenesis, development and prognosis of diseases; (2) the benefits of treatments that control blood viscosity at different stages of disease; and (3) the correlations between hemorheological parameters and other clinical parameters. A general system of diagnosis and monitoring of the effectiveness of treatments should also be established.

Research on blood viscosity has facilitated the development of Biomechanopharmacology [97], a new borderline discipline that considers the effect of pharmacological interventions on biomechanical factors and the biomechanical influence of pharmacokinetics and pharmacodynamics. Me- chanical impacts on endothelial cell functions are an important concern of Biomechanopharmacology. Shear stress results from flow flux and hydro-viscosity, and regulates endothelial cell structure and function; however, the relationship between flow flux and hydro-viscosity has not been comprehensively investigated. Many studies merely focus on changes in shear stress caused by altering the flow flux without considering hydro-viscosity, which is also a major determinant of shear stress [98]. Further intensive studies on blood viscosity will promote the development of Biomechanopharmacology.

Studies of the pathogenesis of hemorrhagic shock and strategies for resuscitation have identified changes in hemorheology during the early stages of hemorrhagic shock [99]. Different plasma volume expanders cause different changes in blood viscosity and the rheological parameters of RBCs, which further affects perfusion of the microcirculation $[100,101]$. In future, we intend to establish both in vitro and in vivo research systems to investigate the mechanisms that regulate blood and plasma viscosity. The influence of blood viscosity on the mechano-properties of endothelial cells will be one focus of our future studies.

This work was supported by the National Natural Science Foundation of China (30800214).

1 Dintenfass L. Rheology of Blood in Diagnostic and Preventive Medicine: An Introduction to Clinical Haemorheology. London, Boston: Butterworths, 1976. 396

2 Liao F L. Clinical Hemorheology (in Chinese). Tianjin: Tianjin Science \& Technology Translation \& Publishing Co, 1987. 210

3 Chen W J. Hemorheology (in Chinese). Tianjin: Tianjin Science and Technology Press, 1987. 322

4 Salazar Vazquez B Y, Martini J, Chavez Negrete A, et al. Cardiovascular benefits in moderate increases of blood and plasma viscosity surpass those associated with lowering viscosity: Experimental and clinical evidence. Clin Hemorheol Microcirc, 2010, 44: 75-85

5 Jeong S K, Cho Y I, Duey M, et al. Cardiovascular risks of anemia correction with erythrocyte stimulating agents: Should blood viscosity be monitored for risk assessment? Cardiovasc Drugs Ther, 2010, 24: $151-160$

6 Allen M T, Patterson S M. Hemoconcentration and stress: A review of physiological mechanisms and relevance for cardiovascular disease risk. Biol Psychol, 1995, 41: 1-27

7 De Simone G, Devereux R, Chien S, et al. Relation of blood viscosity to demographic and physiologic variables and to cardiovascular risk factors in apparently normal adults. Circulation, 1990, 81: 107-117

8 Pehlivanoglu B, Dikmenoglu N, Balkanci D Z. Effect of stress on erythrocyte deformability, influence of gender and menstrual cycle. Clin Hemorheol Microcirc, 2007, 37: 301-308

9 Boer D D, Ring C, Curlett A C, et al. Mental stress-induced hemoconcentration and its recovery: A controlled study of time course and mechanisms. Psychophysiology, 2007, 44: 161-169

10 Galduroz J C F, Antunes H K, Santos R F. Gender- and age-related variations in blood viscosity in normal volunteers: A study of the effects of extract of Allium sativum and Ginkgo biloba. Phytomedicine, 2007, 14: 447-451

11 Gonez C, Donayre M, Villena A, et al. Hematocrit levels in children at sea level and at high altitude: Effect of adrenal androgens. Hum Biol, 1993, 65: 49-57

12 Temte J L. Elevation of serum cholesterol at high altitude and its relationship to hematocrit. Wilderness Environ Med, 1996, 7: 216-224 
13 Bassuni W, Asindi A A, Mustafa F S, et al. Hemoglobin and hematocrit values of Saudi newborns in the high altitude of Abha, Saudi Arabia. Ann Saudi Med, 1996, 16: 527-529

14 Erzurum S C, Ghosh S, Janocha A J, et al. Higher blood flow and circulating NO products offset high-altitude hypoxia among Tibetans. Proc Natl Acad Sci USA, 2007, 104: 17593-17598

15 Cheng S K, Yu J Y, Si B H, et al. Study on the relationship of high hematocrit with changes of the blood viscosity during in the environment of altitude hypoxia (in Chinese). Chin J Appl Physiol, 2001, 17: 231-234

16 Martini J, Carpentier B, Chávez Negrete A, et al. Beneficial effects due to increasing blood and plasma viscosity. Clin Hemorheol Microcirc, 2006, 35: 51-57

17 Faeh D, Gutzwiller F, Bopp M, et al. Lower mortality from coronary heart disease and stroke at higher altitudes in Switzerland. Circulation, 2009, 120: 495-501

18 De Simone G, Devereux R B, Chinali M, et al. Association of blood pressure with blood viscosity in american indians: The Strong Heart Study. Hypertension, 2005, 45: 625-630

19 Salazar Vázquez B Y, Salazar Vázquez M A, Guajardo Jaquez M, et al. Blood pressure directly correlates with blood viscosity in diabetes type 1 children but not in normals. Clin Hemorheol Microcirc, 2010, 44: 55-61

20 Salazar Vázquez B Y, Vazquez M A, Intaglietta M, et al. Hematocrit and mean arterial blood pressure in pre- and postmenopause women. Vasc Health Risk Manag, 2009, 5: 483-488

21 Salazar Vázquez B Y, Intaglietta M, Rodríguez-Morán M, et al. Blood pressure and hematocrit in diabetes and the role of endothelial responses in the variability of blood viscosity. Diabetes Care, 2006, 29: $1523-1528$

22 Loscalzo J. Oxidative stress in endothelial cell dysfunction and thrombosis. Pathophysiol Haemost Thromb, 2002, 32: 359-360

23 Lowe G D, Lee A J, Rumley A, et al. Blood viscosity and risk of cardiovascular events: The Edinburgh Artery Study. Br J Haematol, 1997, 96: 168-173

24 Lee A J, Mowbray P I, Lowe G D, et al. Blood viscosity and elevated carotid intima-media thickness in men and women: The Edinburgh Artery Study. Circulation, 1998, 97: 1467-1473

25 Cecchi E, Marcucci R, Poli D, et al. Hyperviscosity as a possible risk factor for cerebral ischemic complications in atrial fibrillation patients. Am J Cardiol, 2006, 97: 1745-1748

26 Woodward M, Rumley A, Tunstall-Pedoe H, et al. Does sticky blood predict a sticky end? Associations of blood viscosity, haematocrit and fibrinogen with mortality in the West of Scotland. Brit J Haematol, 2003, 122: 645-650

27 Skretteberg P T, Bodegard J, Kjeldsen S E, et al. Interaction between inflammation and blood viscosity predicts cardiovascular mortality. Scand Cardiovasc J, 2010, 44: 107-112

28 Cecchi E, Liotta A A, Gori A M, et al. Relationship between blood viscosity and infarct size in patients with ST-segment elevation myocardial infarction undergoing primary percutaneous coronary intervention. Int J Cardiol, 2009, 134: 189-194

29 Malinova L I, Dovgalevsky P Y. Hemorheological system in coronary heart disease patients: Prognostic value. Cardiovasc Ther Prev, 2007, 6: 19-24

30 Ciuffetti G, Schillaci G, Lombardini R, et al. Prognostic impact of low-shear whole blood viscosity in hypertensive men. Eur J Clin Invest, 2005, 35: 93-98

31 Cohen J D. Overview of physiology, vascular biology, and mechanisms of hypertension. J Manag Care Pharm, 2007, 13: 6-8

32 Baron A D, Clark M G. Role of blood flow in the regulation of muscle glucose uptake. Annu Rev Nutr, 1997, 17: 487-499

33 Hoieggen A, Fossum E, Moan A, et al. Whole-blood viscosity and the insulin-resistance syndrome. J Hypertens, 1998, 16: 203-210

34 Ercan M, Konukoglu D. Role of plasma viscosity and plasma homocysteine level on hyperinsulinemic obese female subjects. Clin Hemorheol Microcirc, 2008, 38: 227-234

35 Richards R S, Nwose E U. Blood viscosity at different stages of diabetes pathogenesis. Br J Biomed Sci, 2010, 67: 67-70

36 Tamariz L J, Young J H, Pankow J S, et al. Blood viscosity and hema- tocrit as risk factors for type 2 diabetes mellitus: The atherosclerosis risk in communities (ARIC) study. Am J Epidemiol, 2008, 168: 1153-1160

37 Lowe G D, Lowe J M, Drummond M M, et al. Blood viscosity in young male diabetics with and without retinopathy. Diabetologia, 1980, 18: 359-363

38 Turczynski B, Michalska-Malecka K, Slowinska L, et al. Correlations between the severity of retinopathy in diabetic patients and whole blood and plasma viscosity. Clin Hemorheol Microcirc, 2003, 29: 129-137

39 Irace C, Scarinci F, Scorcia V, et al. Association among low whole blood viscosity, haematocrit, haemoglobin and diabetic retinopathy in subjects with type 2 diabetes. Br J Ophthalmol, 2011, 95: 94-98

40 Schut N H, van Arkel E C, Hardeman M R, et al. Blood and plasma viscosity in diabetes: Possible contribution to late organ complications? Diabetes Res, 1992, 19: 31-35

41 Zhang X L, Wang X M, Liao L S. The significance of hemorheology changes in patients with diabetic nephropathy (in Chinese). Chin J Nephrol, 1993, 9: 348-349

42 Khodabandehlou T, Vimeux M, Le Devehat C. Measurements of transcutaneous oxygen pressure and changes in blood rheology as markers of prognosis of critically ischemic limb in diabetes mellitus patients. Int J Low Extrem Wounds, 2003, 2: 13-18

43 Cohen R A. Role of nitric oxide in diabetic complications. Am J Ther, 2005, 12: 499-502

44 Le Devehat C, Vimeux M, Khodabandehlou T. Blood rheology in patients with diabetes mellitus. Clin Hemorheol Microcirc, 2004, 30: 297-300

45 Giansanti R, Rabini R A, Boemi M. Blood rheology changes and disturbances in microcirculation. Clin Hernorheol, 1996, 16: 543-548

46 Vigilance J E, Reid H L. Glycaemic control influences peripheral blood flow and haemorheological variables in patients with diabetes mellitus. Clin Hemorheol Microcirc, 2005, 33: 337-346

47 Zanazzi M, Fatini C, Farsetti S, et al. Blood rheology and renal transplantation: An intriguing relationship for assessing cardiovascular risk. Transplant Proc, 2010, 42: 1383-1384

48 Booth S, Chohan S, Curran J C, et al. Whole blood viscosity and arterial thrombotic events in patients with systemic lupus erythematosus. Arthritis Rheum, 2007, 57: 845-850

49 Smith M M, Chen P C Y, Li C S, et al. Whole blood viscosity and microvascular abnormalities in Alzheimer's Disease. Clin Hemorheol Microcirc, 2009, 41: 229-239

50 Chew S H, Tomic M M S, Cheung A T W. Alzheimer's disease: More than amyloid. Clin Hemorheol Microcirc, 2010, 46: 69-73

51 Omoti C E, Omuemu C E, Olu-Eddo A N. The rheological profile of chronic liver disease patients in Nigeria. Clin Hemorheol Microcirc, 2009, 42: 279-284

52 Gokturk H S, Demir M, Ozturk N A, et al. Plasma viscosity changes in patients with liver cirrhosis. South Med J, 2009, 102: 1013-1018

53 Fang N, Jiang L X, Zhang X S. Clinical observation of blood viscosity in patients with viral hepatitis (in Chinese). Chin J Hemorh, 2000, 10: $240-241$

54 Halis H, Bor-Kucukatay M, Akin M, et al. Hemorheological parameters in children with iron-deficiency anemia and the alterations in these parameters in response to iron replacement. Pediatr Hematol Oncol, 2009, 26: 108-118

55 Zhao L, Wang B, You G X, et al. Effects of different resuscitation fluids on the rheologic behavior of red blood cells, blood viscosity and plasma viscosity in experimental hemorrhagic shock. Resuscitation, 2009, 80: 253-258

56 Wettstein R, Erni D, Intaglietta M, et al. Rapid restoration of microcirculatory blood flow with hyperviscous and hyperoncotic solutions lowers the transfusion trigger in resuscitation from hemorrhagic shock. Shock, 2006, 25: 641-646

57 Wettstein R, Tsai A G, Erni D, et al. Improving microcirculation is more effective than substitution of red blood cells to correct metabolic disorder in experimental hemorrhagic shock. Shock, 2004, 21: 235-240

58 Zhao L, You G X, Zhou H. High viscosity plasma expander is beneficial for resuscitation of hemorrhagic shock (in Chinese). J Int Pharm Res, 2008, 35: 360-363

59 Kilpatrick D, Fleming J, Clyne C, et al. Reduction of blood viscosity 
following plasma exchange. Atherosclerosis, 1979, 32: 301-306

60 Brown M M, Marshall J. Effect of plasma exchange on blood viscosity and cerebral blood flow. Br Med J (Clin Res Ed), 1982, 284: 1733-1736

61 Cliville X, Bofill C, Joven J, et al. Hemorheological, coagulative and fibrinolytic changes during autologous blood donation. Clin Hemorheol Microcirc, 1998, 18: 265-272

62 DeFilippis A P, Law K, Curtin S, et al. Blood is thicker than water: The management of hyperviscosity in adults with cyanotic heart disease. Cardiol Rev, 2007, 15: 31-34

63 Strand T, Asplund K, Eriksson S, et al. A randomized controlled trial of hemodilution therapy in acute ischemic stroke. Stroke, 1984, 15: 980-989

64 Ferrannini M, Vischini G, Staffolani E, et al. Rheopheresis in vascular diseases. Int J Artif Organs, 2007, 30: 923-929

65 Dou M, Ma A G, Wang Q Z, et al. Supplementation with magnesium and vitamin $\mathrm{E}$ were more effective than magnesium alone to decrease plasma lipids and blood viscosity in diabetic rats. Nutr Res, 2009, 29: 519-524

66 Chung T W, Yu J J H, Liu D Z. Reducing lipid peroxidation stress of erythrocyte membrane by alpha-tocopherol nicotinate plays an important role in improving blood rheological properties in type 2 diabetic patients with retinopathy. Diabet Med, 1998, 15: 380-385

67 Feng X L, Jin Y H, Lin J H. Effects of the purified oil of Anguilla japonica and vitamin $\mathrm{E}$ on the concentration of blood fibrinogen and the blood viscosity of rat (in Chinese). Acad J First Med Coll PLA, 1999, 19: 122-123

68 Bin J P, Doctor A, Lindner J, et al. Effects of nitroglycerin on erythrocyte rheology and oxygen unloading-Novel role of $S$-nitrosohemoglobin in relieving myocardial ischemia. Circulation, 2006, 113: 2502-2508

69 Hou W C, Tsay H S, Liang H J, et al. Improving abnormal hemorheological parameters in aging guinea pigs by water-soluble extracts of Salvia miltiorrhiza Bunge. J Ethnopharmacol, 2007, 111: 483-489

70 Fan H Y, Fu F H, Yang M Y, et al. Antiplatelet and antithrombotic activities of salvianolic acid A. Thromb Res, 2010, 126: 17-22

71 Tian J W, Fu F H, Jiang W L, et al. Protective effect of Ligulsticum chuanxiong phthalides on focai cerebral ischemia in rats and its related mechanism of action. Zhongguo Zhongyao Zazhi, 2005, 30: 466-468

72 Naito T, Kubota K, Shimoda Y, et al. Effects of constituents in a Chinese crude drug, Ligustici chuanxiong Rhizoma on vasocontraction and blood viscosity. Nat Med, 1995, 49: 288-292

73 Liu J X, Li H Y, Mu Y J, et al. The effect of Qigen Xingnao Prescriptionon on platelet aggregation, thrombosis and hemorrheology (in Chinese). Chin Tradit Pat Med, 2007, 30: 756-758

74 Salazar Vquez B Y, Wettstein R, Cabrales P, et al. Microvascular experimental evidence on the relative significance of restoring oxygen carrying capacity vs. blood viscosity in shock resuscitation. Biochim Biophys Acta, 2008, 1784: 1421-1427

75 Cabrales P, Intaglietta M, Tsai A G. Transfusion restores blood viscosity and reinstates microvascular conditions from hemorrhagic shock independent of oxygen carrying capacity. Resuscitation, 2007, 75: $124-134$

76 Cabrales P, Tsai A G, Intaglietta M. Is resuscitation from hemorrhagic shock limited by blood oxygen-carrying capacity or blood viscosity? Shock, 2007, 27: 380-389

77 Martini J, Tsai A G, Cabrales P, et al. Increased cardiac output and microvascular blood flow during mild hemoconcentration in hamster window model. Am J Physiol Heart Circ Physiol, 2006, 291: 310-317

78 Hess J R, MacDonald V W, Brinkley W W. Systemic and pulmonary hypertension after resuscitation with cell-free hemoglobin. J Appl Physiol, 1993, 74: 1769-1778

79 Keipert P E, Gonzales A, Gomez C L, et al. Acute changes in systemic blood pressure and urine output of conscious rats following exchange transfusion with diaspirin-crosslinked hemoglobin solution. Transfusion, 1993, 33: 701-708

80 Hess J R, MacDonald V W, Winslow R M. Dehydration and shock:
An animal model of hemorrhage and resuscitation of battlefield injury. Biomater Artif Cells Immobilization Biotechnol, 1992, 20: 499-502

81 Caron A, Malfatti E, Aguejouf O, et al. Vasoconstrictive response of rat mesenteric arterioles following infusion of cross-linked, polymerized, and conjugated hemoglobin solutions. Artif Cells Blood Substit Immobil Biotechnol, 2001, 29: 19-30

82 Winslow R M, Gonzales A, Gonzales M I, et al. Vascular resistance and the efficacy of red cell substitutes in a rat hemorrhage model. J Appl Physiol, 1998, 85: 993-1003

83 Rochon G, Caron A, Toussaint-Hacquard M, et al. Hemodilution with stoma-free hemoglobin at physiologically maintained viscosity delays the onset of vasoconstriction. Hypertension, 2004, 43: 1110-1115

84 Tsai A G, Acero C, Nance P R, et al. Elevated plasma viscosity in extreme hemodilution increases perivascular nitric oxide concentration and microvascular perfusion. Am J Physiol Heart Circ Physiol, 2005, 288: 1730-1739

85 Tsai A G, Friesenecker B, McCarthy M, et al. Plasma viscosity regulates capillary perfusion during extreme hemodilution in hamster skinfold model. Am J Physiol Heart Circ Physiol, 1998, 275: 2170-2180

86 Cabrales P, Tsai A G, Intaglietta M. Increased plasma viscosity prolongs microhemodynamic conditions during small volume resuscitation from hemorrhagic shock. Resuscitation, 2008, 77: 379-386

87 Tok D, Caliskan M, Gullu H, et al. The association between hematological parameters and coronary flow reserve and coronary haemorheology in healthy subjects. Clin Hemorheol Microcirc, 2007, 36: 345-352

88 Starzyk D, Korbut R, Gryglewski R J. Effects of nitric oxide and prostacyclin on deformability and aggregability of red blood cells of rats ex vivo and in vitro. J Physiol Pharmacol, 1999, 50: 629-637

89 Bor-Kucukatay M, Wenby R B, Meiselman H J, et al. Effects of nitric oxide on red blood cell deformability. Am J Physiol Heart Circ Physiol, 2003, 284: 1577-1584

90 Dangel O, Mergia E, Karlisch K, et al. Nitric oxide-sensitive guanylyl cyclase is the only nitric oxide receptor mediating platelet inhibition. J Thromb Haemost, 2010, 8: 1343-1352

91 Malek A, Izumo S. Physiological fluid shear stress causes downregulation of endothelin-1 mRNA in bovine aortic endothelium. Am J Physiol-Cell Ph, 1992, 263: 389-396

92 Koller A, Sun D, Kaley G. Role of shear stress and endothelial prostaglandins in flow- and viscosity-induced dilation of arterioles in vitro. Circ Res, 1993, 72: 1276-1284

93 Bertuglia S. Increased viscosity is protective for arteriolar endothelium and microvascular perfusion during severe hemodilution in hamster cheek pouch. Microvasc Res, 2001, 61: 56-63

94 Byfield F J, Reen R K, Shentu T P, et al. Endothelial actin and cell stiffness is modulated by substrate stiffness in 2D and 3D. J Biomech, 2009, 42: 1114-1119

95 Chowdhury F, Li Y Z, Poh Y C, et al. Soft substrates promote homogeneous self-renewal of embryonic stem cells via downregulating cell-matrix tractions. PLoS One, 2010, 5: e15655

96 Tee S Y, Fu J P, Chen C S, et al. Cell shape and substrate rigidity both regulate cell stiffness. Biophys J, 2011, 100: 25-27

97 Liao F L, Li M, Han D, et al. Biomechanopharmacology: A new borderline discipline. Trends Pharmacol Sci, 2006, 27: 287-289

98 Chiu J J, Chien S. Effects of disturbed flow on vascular endothelium: Pathophysiological basis and clinical perspectives. Physiol Rev, 2011, 91: 327-387

99 Zhao L, Wang B, You G X, et al. Hemorrheological changes in irreversible hemorrhagic shock (in Chinese). Chin Crit Care Med, 2008, 20: $159-162$

100 Zhao L, Wang B, You G X, et al. Effects of different resuscitation fluids on the rheologic behavior of red blood cells, blood viscosity and plasma viscosity in experimental hemorrhagic shock. Resuscitation, 2009, 80: 253-258

101 Zhao L, You G X, Liao F L, et al. Sodium alginate as viscosity modifier may induce aggregation of red blood cells. Artif Cells Blood Substit Immobil Biotechnol, 2010, 38: 267-276

Open Access This article is distributed under the terms of the Creative Commons Attribution License which permits any use, distribution, and reproduction in any medium, provided the original author(s) and source are credited. 